\title{
Taking away PAIN: Enabling new structures of the Nav1.7 sodium channel by cryo-EM \\ C Jao ${ }^{1}$, C Arthur ${ }^{2}$, A Rohou ${ }^{3}$, M Kschonsak ${ }^{4}$, D Ortwine ${ }^{5}$, B Sellers ${ }^{6}$, P Dragovich ${ }^{7}$, Ma Volgraf ${ }^{8}$,

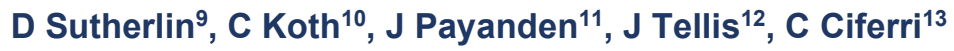 \\ ${ }^{1}$ Genentech ${ }^{2}$ Genentech, ${ }^{3}$ Genentech, ${ }^{4}$ Genentech, ${ }^{5}$ Genentech, ${ }^{6}$ Genentech, ${ }^{7}$ Genentech, ${ }^{8}$ Genetech, ${ }^{9}$ Genetech, ${ }^{10}$ Genetech, ${ }^{11}$ Genetech, ${ }^{12}$ Genetech \\ ${ }^{13}$ Genetech \\ jao.christine@gene.com
}

\begin{abstract}
Mutations in human voltage-gated sodium channels are associated with a range of neurological and cardiovascular diseases. For example, loss-of-function mutations in Nav1.7 cause a striking congenital insensitivity to pain, whereas gain-of-function mutations are associated with persistent pain disorders. These opposing phenotypes define Nav1.7 as integral to pain perception and have motivated intense efforts to design therapeutics that selectively and potently inhibit its activity. Previously, we reported that the aryl sulfonamide class of Nav1.7 antagonists of Nav1.7 bind to the activated state of voltage sensor domain IV (VSD4), inhibiting the channel through a voltage-sensor trapping mechanism to oppose channel deactivation. In this talk, I will present our establishment of a new, robust system for the determination of many additional high-resolution structures of Nav1.7 bound to other small molecule series, the unexpected mechanism of action of the acyl sulfonamide class of antagonists, and the use of these structures to guide the design of a new 'hybrid' class of inhibitors.
\end{abstract}

